\title{
Optimal Design and Software Development of Two-stage NGW Planetary Transmission
}

\author{
Yong $\mathrm{Gu}^{1, \text { a }}$, Siming Luo ${ }^{1, \mathrm{~b}}$, Tingting Yang ${ }^{1, \mathrm{c}}$, Jiawei Wang ${ }^{1, \mathrm{~d}}$, Fugui Zhu ${ }^{2, \mathrm{e}}$ \\ ${ }^{1}$ Logistics Engineering, Wuhan University of Technology, Wuhan, China; \\ ${ }^{2}$ South China University of Technology, Guangzhou, China \\ a108789742@qq.com, b465260856@qq.com, '373622800@qq.com, d1793564897@qq.com \\ eziqianedu@qq.com
}

Keywords: Planetary Transmission; Optimal Design; Software Development.

\begin{abstract}
Study of NGW type planetary gear transmission problem, and establish transmission model and mathematical model according to the conditions of planetary transmission, then use the theory of minimizing the objective function to complete the target function, develop the corresponding optimization design program of user interface and optimization algorithm for semi random discrete variable complex method based on the Visual Object-Oriented high-level programming language by $\mathrm{C \#}$ and VBA, complete the optimization design of Two-stage NGW Planetary Transmission.
\end{abstract}

\section{Introduction}

Planetary gear transmission system is widely used in engineering machinery, automotive, aerospace and other all kinds of mechanical transmission which having a high requirement of the field because of many advantages and high performance. Optimization of planetary transmission mechanism has experienced from the initial single stage transmission, low dimensional variable optimization to now the multistage transmission, high dimension optimization, from classical optimization algorithm to various intelligent exploratory put forward and application of optimization algorithm, fuzzy design, the transformation from the traditional design to the robustness of design thought, all of these provide a good foundation of planetary gear reducer with accurate rapid design and manufacture.

Optimization design for planetary transmission mechanism, optimization are mainly concentrated in the main structure parameters of gear. From the beginning of the 80's of last century, the application of computer to promote the optimization of our planetary transmission design research development, in the process of development, with the emergence of various optimization algorithms and commercial design software and optimization toolbox for optimization design, provided the conditions for planetary reducer.

\section{Design and model of two stage NGW Planetary Transmission}

Establish the optimization model according to the conditions of planetary transmission system strength condition, performance conditions and assembly condition by minimizing the volume of two-stage planetary gear transmission system. Figure 1 is physical model of planetary gear, figure 2 is the Simplified transmission of planetary gear. The first stage outer meshing center wheel A1 is connected with a motor and the first stage planetary frame X1 is connected with the second grade external meshing center wheel A1, the second planetary frame X2 is connected with the output shaft and the load. 


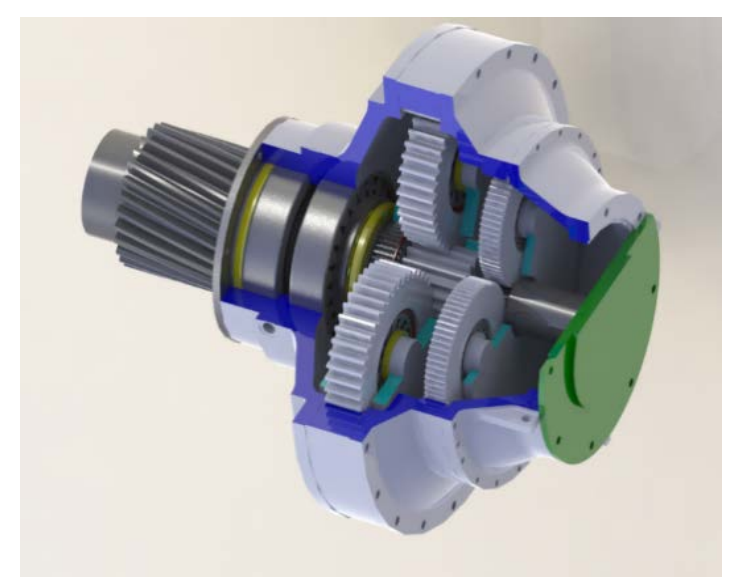

Fig.1 Physical model of planetary gear

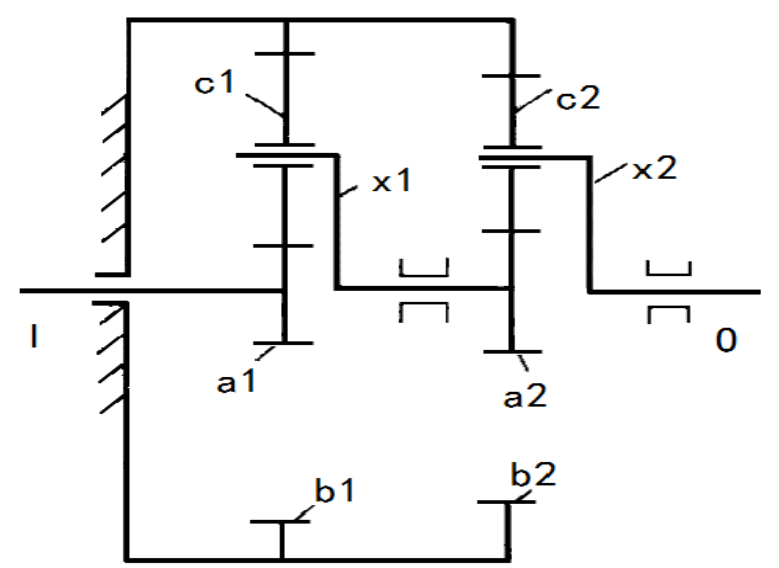

Fig.2 Simplified transmission of planetary gear

\section{The design variables and objective function}

The design variables are variable parameters can influence the quality of design and results, and for planetary gear system by taking minimum volume as the target of optimization, the main 15 parameters are related to transmission system volumes, they are: number of teeth $z_{a}, z_{b}, z_{\varepsilon}$, and the modulus $\mathrm{m}$, tooth width $\mathrm{b}$, displacement coefficient $x_{\mathbb{a}}, x_{a}, x_{a}$, theory of center distance $a_{a \varepsilon}, a_{b c}$ and the actual center distance $a_{a c}^{t}$, meshing angle $\alpha_{a c}, \alpha_{a c}$ and installation coefficient $\mathrm{w}$.

Installation conditions:

$z_{a}+z_{b}=n_{p} \cdot W$

Geometric dimension relations:

$$
\begin{aligned}
& a_{a c} \cdot \cos \alpha_{0}=a_{a c}{ }^{\prime} \cdot \cos \alpha_{a c} \\
& a_{b c} \cdot \cos \alpha_{0}=a_{b c} \cdot \cos \alpha_{b c} \\
& a_{a c}=\frac{m}{2}\left(z_{a}+z_{c}\right) \\
& a_{b c}=\frac{m}{2}\left(z_{b}-z_{c}\right)
\end{aligned}
$$

Concentric condition:

$$
a_{a c}{ }^{\prime}=a_{b c}{ }^{\prime}=a^{\prime}
$$

Non backlash meshing condition:

$$
\begin{aligned}
& x_{a}+x_{c}=\frac{z_{a}+z_{c}}{2 \tan \alpha_{0}}\left(\operatorname{inv} \alpha_{a c}-\operatorname{inv} \alpha_{0}\right) \\
& x_{b}+x_{c}=\frac{z_{b}-z_{c}}{2 \tan \alpha_{0}}\left(\operatorname{inv} \alpha_{b c}-\operatorname{inv} \alpha_{0}\right)
\end{aligned}
$$

Based on the theory of linear programming, all the values of the variables can be completed by only 7 independent variables. And when deal with equality constraints, usually considering many optimization algorithm, then transform equality constraints into inequality constraints by using the threshold of a minimum constant. In this paper, the parameters of deformation $z_{b}$ is processed properly when setting the design variables, then introduce a determined by installing constraint coefficient $W=\left(z_{a}+z_{b}\right) / n_{p}$, it can improve the properties of the feasible region effectively, so it's easier to solve the optimal solution of objective function.

Considering design variables discretization, round of optimization results, improve the feasible domain, the constraint functions and other issues, so conclude the design variables as:

$$
X=\left[m, z_{a}, z_{c}, W, \mathrm{~b}, a, \mathrm{x}_{a}\right]
$$

Consider the parts and many parameters reducer volume involved, different scholars deal with these problems, although not a choice, but is in gear reducer volume and volume as measured by 
standard. So when material is certain, In order to make the minimum weight design of reducer, that is to make the smallest volume. In this paper, the optimization measure is according to the standard spur gear addendum circle calculation speed reducer volume.

Now establish the structural optimization design of planetary gear reducer, with minimize the objective function and the volume of the sun gear and the planet wheels objective. Among them, in the calculation of volume, cross-sectional area of gear according to the standard spur gear addendum circle area calculation. So the target function is

$$
V=\frac{\pi}{4} \cdot\left[\mathrm{m}_{1}^{2} \mathrm{~b}_{1}\left(\mathrm{z}_{a 1}^{2}+n_{p} \cdot \mathrm{z}_{c 1}^{2}\right)+\mathrm{m}_{2}^{2} \mathrm{~b}_{2}\left(\mathrm{z}_{a 2}^{2}+n_{p} \cdot \mathrm{z}_{c 2}^{2}\right)\right]
$$

\section{Development of NGW application software of planetary gear train}

Develop the user interface development optimization algorithm for semi random discrete variable complex method and the correspond optimization design program based on Visual Studio and Excel as the basic development environment with $\mathrm{C \#}$ and VBA in a visual object oriented high-level programming language.

Now know the star reducer output torque $\mathrm{T}=4 \mathrm{~T} \cdot \mathrm{S}$; motor input power $\mathrm{P}=75 \mathrm{KW}$, input speed $\mathrm{n}=1000 \mathrm{r} / \mathrm{min}$; allow the transmission ratio error of the $\mathrm{i}=0.01$; everyday works 8 hours, and 300 days a year, the required service life of 8 years, input interface parameters and see figure 3 in Figure 4, by software calculation, we obtain the optimum design results from Figure 5.

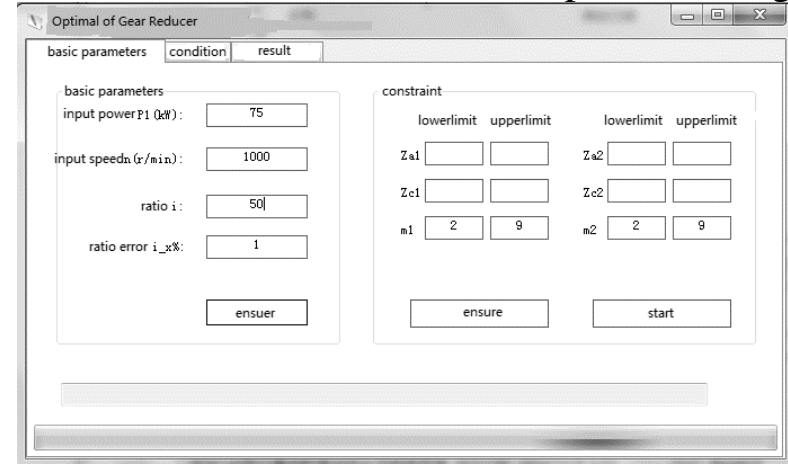

Fig.3 Input basic parameters

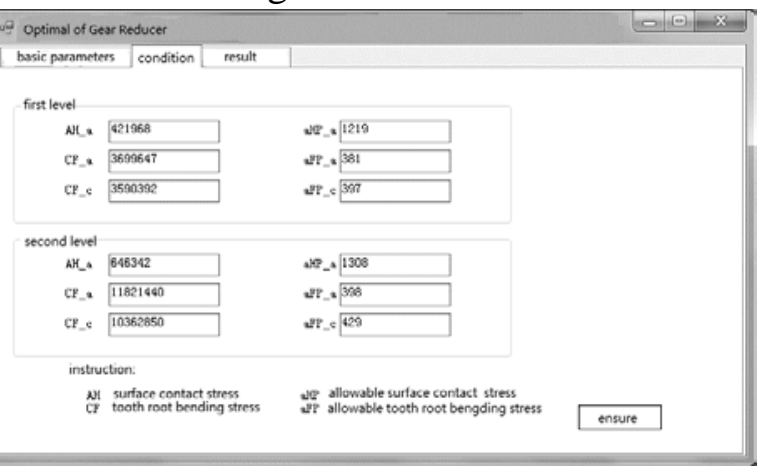

Fig.4 Input condition

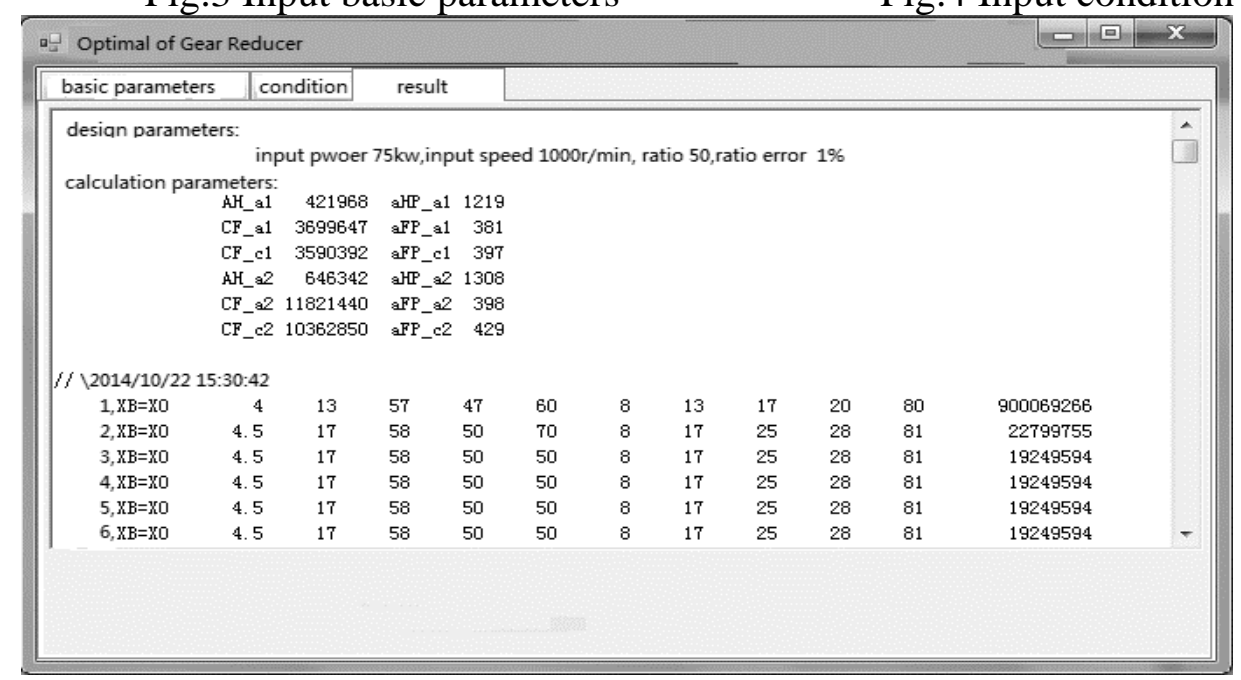

Fig.5 Results output

\section{Conclusion}

Design parameters of transmission system of NGW type planetary gear Optimization toolbox to optimize the design of NGW type planetary gear transmission mechanism in this paper, select the appropriate combination of , then set up design parameter optimization design problem, write the corresponding program optimization based on the traditional complex shape method effective 
objective function in the design of space non convex characteristics and the complex method of mixed discrete variables commonly used in engineering, then solve practical example optimization. The optimization results show that the optimization algorithm in this paper compared with the traditional design method and the composite shape simple method is more effectively.

\section{Acknowledgments}

Supported by "the Fundamental Research Funds for the Central Universities(WUT:2014-IV-146)"

\section{References}

[1] Rao S. S: Description and Optimum Design of Fuzzy Mechanical Systems [J]. ASME Journal of Mechanisms Transmissions and Automation in Design, 1987, 109(1), p.126-132.

[2] E. M. Al-Shareedah, H. Alawi: Reliability analysis of bevel gears with and without back support [J]. Mechanism and Machine Theory, 1987, 22(1), p.13-20.

[3] Rajeev S, Krishnamurthy C S: Discrete optimization of structures using genetic algorithm[J]. Journal of structural engineering, 1992, 11(8), p.1233-1250.

[4] Kyung-Eun Ko, Do-Hyeong Lim, etc.: A study on the bending strength of the hollow sun gear in a planetary gear train [J]. Journal of Mechanical Science and Technology, 2010, 24, p.29-32. 